compliant and which also contained other salient information relating to the use of strong opioids. The PIL is titled "Morphine and other opioid painkillers for moderate to severe pain" and contains the following section headings:

- Using Opioids - why prescribed, preparation and how to take, managing breakthrough pain

- Side effects - common side effects, what to do if experiencing side effects

- Driving and Travelling

- Other Information - collecting from pharmacy, opioids and alcohol, storage, disposal

There is also the facility on the front cover of the PIL for the dispensing pharmacy to stick duplicate labels of strong opioid medicines dispensed so that patients and carers know to which drugs the PIL refers. The PIL produced has, if locally adapted, wide potential for use in any palliative care organisation and pharmacy dispensing strong opioids for moderate to severe pain in the UK.

\section{P113 RE-DESIGNING HOW PATIENT MEDICINES INFORMATION IS PRESENTED ON DISCHARGE}

${ }^{1,2}$ Ray Bunn, ${ }^{2}$ Amanda Gregory. ${ }^{1}$ Kamson's Pharmacy, Crawley, UK, ${ }^{2}$ St. Catherine's Hospice, Crawley, UK

\subsection{6/bmjspcare-2013-000591.135}

A plethora of written patient information accompanies medicines dispensed (TTOs) for patients discharged from an independent Hospice inpatient unit. This includes:

- Manufacturer's patient information leaflets (PILs) for each medicine used within license - legally required.

- Locally developed PILs for medicines that are unlicensed or for use off-label.

- Green (regular) and blue (when necessary) 'Drug Information Cards' listing all medicines and dosage information electronically transposed from the TTO prescription.

- Locally developed PILs informing about medicine groups warranting special care in use e.g. 'morphine and other opioid painkillers for moderate to severe pain'.

It was observed that this 'jumble' of leaflets and medicines looked disorderly and unprofessional, with a real risk of information being lost, overlooked or simply discarded as unimportant. The aim was to develop how written patient information was presented to patients and carers in a way that was:

- Professional and distinctly noticeable.

- Neat, orderly and clear.

This problem was presented to the hospice Medicines Management Group (MMG) for resolution. The MMG is a multidisciplinary team comprising of medical, nursing and pharmacy representation with roles including the continuously improvement of the quality of pharmaceutical related practice and processes, often in an innovative way. They developed the following solution:

- 'Hospice branded' A4 front and rear cover, cardboard folder with rear cover hole punched near spine.

- Flat bar file fastener fitted to the rear page to hold:

- Explanatory page.

- A4 unlicensed PILs.

- A4 Drug Information Cards.
- An A4 plastic pouch holding the manufacturers PILs (variable sizes)

- The completed file is then placed in the bag containing TTOs.

This solution has potential for application in other settings.

\section{P114 IMPROVING THE USE OF PATIENTS OWN MEDICATION IN THE HOSPICE SETTING}

${ }^{1,2}$ Jeanette Crowther, ${ }^{1,2}$ Steven Wanklyn, ${ }^{1}$ Mel Johnson, ${ }^{1}$ Samantha Lund. ${ }^{1}$ Trinity Hospice, Clapham, London, ' Guy's \& St Thomas' NHS Foundation Trust London

\subsection{6/bmjspcare-2013-000591.136}

Introduction The use a of patients' own medications is improved at Trinity Hospice by patient's being encouraged to bring them in when being admitted. Advantages of using a patient's own medications are widely recognised and include aiding prompt completion of medicines reconciliation, thus achieving more accurate prescribing on admission, medication being available faster on the wards and of a quality that is suitable for self-administration, reducing the number of late and missed doses, patients continuing to use medication brands with which they are familiar or are required to due to inter-brand differences in bioavailability and considerable savings for the hospice's drug expenditure.

Aims

- To establish a procedure framework that improves the re-use of a patient's own medications whilst minimising patient risk.

- To inform governance priorities for the re-use of a patient's own medications particularly those involving high risk drugs.

Method A multidisciplinary team was established with representation from pharmacy, doctors and nurses in order to develop a clinical protocol for the management of a patient's own medications.

The protocol manages how a patient's own medications are assessed for suitability to be administered using a decision-support algorithm, obtaining further supplies and the essential documentation. Staff were deemed competent to undertake this procedure provided they attended a training workshop and successfully complete the training competency.

Results \& Discussion In order to quality-assure the protocol adherence to the process will be audited on a regular basis. The results of this alongside incident surveillance will provide ongoing risk management and identify areas for improvement.

Conclusion Trinity hospice has approved this protocol. Staff eligible to undertake a role have been accredited to do so. Under the terms of the Hospice's approval an audit of adherence and critical risk assessment will inform the further development of this medicine system.

\section{P115 CLINICAL PHARMACISTS - HANDS ON IN THE PATIENTS HOME AT END OF LIFE}

Joanne Bartlett, Louise Seager. John Taylor Hospice CIC, Birmingham, UK

\subsection{6/bmjspcare-2013-000591.137}

The introduction of frontline clinical pharmacy services for patients in their preferred place of care is redefining the 
traditional role of the specialist clinical pharmacist in palliative care.

This innovative role allows patients, carers and other healthcare professions to access the expertise of the clinical pharmacist in the patients home and is aimed specifically at complex patient groups moving towards end of life.

The aim of the role is to enable patients with more complex drug needs to remain in their preferred place of care, the role sits in a wider specialist community team comprising of physiotherapists, occupational therapists, dieticians, social worker and nurses.

Referrals include

- Complex symptom control i.e. unresponsive to or having experienced side effects and adverse drug reactions with conventional first and second line therapies

- Patients with multiple co-morbidities on complex regimes who require rationalisation of their medicines

- Patients requiring medication review/rationalisation including following discharge from secondary care

- Drug/dose choice for patients with impaired drug metabolism or clearance i.e. renal/liver impairment

- Advice on drug choice, route and formulation for patients with drug administration difficulties

- Issues around access to specialist palliative care medicines in the community setting

- Patients with a non-cancer diagnosis including those with end stage heart failure, renal failure, COPD, liver failure, motor neurone disease etc.

- Patients on palliative chemotherapy

In addition to working with the specialist palliative care team there are many external links these include GPs, district nurses, specialist clinical nurses (community and hospital), consultants, community pharmacists and hospice at home to provide seamless care for palliative patients across the interface. This involves joint professional visits, attendance of GSF meetings or hospital MDT meetings etc.

The Clinical Pharmacy team prescribe for their patients ensuring timely access to specialist medicines and prompt symptom control

\section{P116 ANTICIPATORY MEDICATION 'AS STOCK' FOR RESIDENTS WHO ARE IN THE DYING PHASE: A PROJECT IN 3 NURSING CARE HOMES}

Kathy Morris, Jo Hockley, Margaret Gibbs. St. Christopher's Hospice, London, UK

\subsection{6/bmjspcare-2013-000591.138}

The Gold Standards Framework for Care Homes [GSFCH] and the Liverpool Care Pathway [LCP] guidance suggests the importance of obtaining anticipatory medication for the control of symptoms in the last days of life for nursing home $(\mathrm{NH})$ residents. There is considerable wastage however as NH residents are dispensed anticipatory drugs on a named-patient basis. There is also evidence that when these drugs are not available residents are hospitalised inappropriately.

Aim The aims of the project were to explore the frequency of symptoms experienced in the dying phase and to explore whether there was a need for residents to have their own supply of drugs by examining wastage. The idea was to establish a process in order for homes to obtain anticipatory medication as 'stock' and to capture the benefits of doing this.

Process The managers of three NHs, who had shown interest in obtaining medication for the last days of life, met with a local
GSFCH facilitator, specialist palliative care pharmacist and a pharmacist from the regulatory body. A proposal that included a list of necessary medication and how to acquire them was written. The NH staff developed the required Standard Operating Procedures. A prospective audit on medication used was commenced.

Results Significant improvements in the availability of anticipatory medication were found. $53 \%$ of residents were symptomatic highlighting the need to have anticipatory medication available. No medication other than "stock" was required by NHs. If all residents had their own supply of medication $£ 4,506$ worth of drugs would have been wasted. All staff involved perceived that this work had been beneficial.

Conclusion Anticipatory medication 'as stock' for people dying in $\mathrm{NHs}$ is an important step forward. There is less wastage of medication, less delay in controlling symptoms, reduced call out of GPs and less anxiety.

\section{P117 THE IMPLEMENTATION OF A LOCALLY DEVELOPED ADVANCE CARE PLANNING DOCUMENT ACROSS FOUR DIVERSE HEALTH AND SOCIAL CARE SETTINGS}

Debbie Dempsey, Sarah Owens. Wigan and Leigh Hospice, Wigan, England

\subsection{6/bmjspcare-2013-000591.139}

Advance care planning (ACP) has seen its profile raised in recent years, with various UK government publications serving to drive forward the importance of identifying people's preferences, wishes and expectations for their future care.

Clear documentation is essential for effective sharing of information amongst the multi-disciplinary team, in a bid to ensure an individuals plan is communicated, respected and goals of care are achieved.

In the author's geographical area, it has been identified that there is no robust ACP document currently in use, particularly within the care home setting, therefore the author facilitated the production of a locally produced ACP document via the local palliative and end of life care strategy group.

This qualitative study used mixed methods including semistructured interviews, questionnaires and a focus group to explore the introduction of this ACP document, highlighting both staff and patient's/residents perceptions of the process and the identified challenges and benefits. Four sites were chosen to pilot the document, (local nursing home, day hospice unit, memory service, supported living complex) representing a diverse range of health and social care services.

Key themes emerged from the analysis of the interviews and questionnaires: Staff confidence, timing of discussions, patient empowerment, patient diagnosis and apprehension regarding legally binding decisions. The perceived importance of having ACP discussions was the underlying similarity between the services; however the challenges varied depending upon the nature of the setting.

The results identified that $23 \mathrm{ACP}$ documents were completed in the pilot phase, with $80 \%$ of patients/residents stating that they would recommend this process to others. Reduction of anxiety, peace of mind and control were frequently expressed by participants.

A need for further research in those diagnosed with early dementia is indicated. Collaboration across services may be needed to develop ACP trigger guidelines that fit the complex needs of diverse patient/client groups. Also the challenge of 